\title{
Proposed score for occlusal-supporting ability
}

\author{
Yasuhiko Abe", Keisuke Nogami, Wataru Mizumachi, Hiroki Tsuka, Kyou Hiasa \\ Department of Advanced Prosthodontics, Applied Life Sciences, Institute of Biomedical \& Health Sciences, Hiroshima University, \\ Hiroshima, Japan \\ Email: ${ }^{\text {abey@hiroshima-u.ac.jp }}$
}

Received 20 April 2013; revised 21 May 2013; accepted 9 June 2013

Copyright (C) 2013 Yasuhiko Abe et al. This is an open access article distributed under the Creative Commons Attribution License, which permits unrestricted use, distribution, and reproduction in any medium, provided the original work is properly cited.

\begin{abstract}
In our previous studies, we developed the normal periodontal ligament index (nPLI) and residual periodontal ligament index (rPLI) to estimate residual periodontal ligament support for individual teeth during treatment planning for partially edentulous patients. To illustrate the applicability of the nPLI and rPLI, an occlusal-supporting ability (OSA) score calculated using these indices for the remaining teeth corresponding to Eichner's subclasses of partial edentulism was charted by numerically assessing the average occlusal support. This OSA score based on the nPLI and rPLI is proposed as a suitable tool for epidemiologic research on the progression of tooth loss and the survival of prostheses. The Ethics Committee for Epidemiologic Study of Hiroshima University approved the study protocol (No. 331).
\end{abstract}

Keywords: Occlusal Support; Ability Score; Periodontal Ligament

\section{INTRODUCTION}

Clinical assessment of the occlusal support of any residual dentition is an integral part of prosthodontic treatment planning. Consideration of the occlusal-supporting abilities (OSAs) of remaining teeth is closely related to the clinical assessment of their physiologic and pathologic periodontal tissue support. The support status is evaluated clinically by measuring pocket depth, attachment level, and tooth mobility, as well as by assessing intraoral radiographs. In particular, the clinical attachment level is determined by probing, and the value is defined as the distance from the cementoenamel junction to the site of an inserted probe tip [1]. Measurement of attachment level can be combined with radiographic assessment of the distance between the cementoenamel junction and the alveolar bone crest to clinically assess

"Corresponding author. abutment teeth for fixed and removable partial dentures.

The crown-to-root ratio or periodontal tissue support is determined and evaluated by Ante's law (1926), which postulates that in fixed partial denture design the total periodontal ligament area of the abutment teeth should be equal to or greater than that of the teeth to be replaced [2]. Furthermore, the length of the periodontal ligament attachment of the abutment tooth should be at least onehalf to two-thirds of its normal attachment [3]. Hence, the estimation of the periodontal ligament area pro- vides useful information for the prognosis of a tooth and its OSA.

When designing fixed and removable partial dentures, the occlusal support of the remaining teeth is usually assessed on the assumption that these teeth have normal optimal periodontal ligament support [3]. However, the residual periodontal ligament area is not usually assessed when determining occlusal support. Yamamoto et al. [4] demonstrated that formulae derived for estimating the residual root surface area attached to the periodontal ligament for each tooth type can be used to assess tooth prognosis along with other factors such as mobility, oral hygiene, degree of inflammation, and occlusion. We previously developed an index for estimating residual periodontal ligament support and the corresponding occlusal support according to tooth type by applying these formulae [5]. The residual periodontal ligament index (rPLI) was derived from a formula that calculates the remaining area of periodontal attachment and the normal periodontal ligament index (nPLI) value, the latter of which assesses average residual OSA. To verify the suitability of this index for prosthodontic treatment planning such as selecting abutment teeth, establishing criteria for tooth extraction, choosing implants, and determining the optimal extent of the denture base areas, it is necessary to accumulate fundamental clinical data about the OSA of each tooth type. Thus, we analyzed the occlusal force, area, and pressure of individual maxillary and mandibular teeth and assessed their OSAs. We concluded that the occlusal pressure of individual teeth 
can be used as an indicator of OSA [6].

Therefore, based on our previous studies [5-8], a score based on nPLI and rPLI values is proposed to estimate the OSA of the remaining teeth.

\section{MATERIALS AND METHODS}

\section{1. nPLI and rPLI of Each Tooth Type}

In a previous study [5], nPLI and rPLI values for each individual tooth were developed to quantitatively assess the average occlusal support (Table 1). Each nPLI value was calculated as the ratio of the tooth's normal periodontal ligament area relative to the entire normal periodontal ligament area of the arch (calculated for 14 unilateral maxillary and mandibular teeth). The total nPLI value for the 14 unilateral teeth was determined to be 50, with a bilateral score of 100 . Therefore, the working formula was:

$\mathrm{nPLI}=[($ individual tooth's normal periodontal ligament area/3869.7) $\times 100] / 2$

According to Yamamoto et al. [4], the residual periodontal ligament area percentage for each tooth $(\mathrm{Y})$ can be calculated as: $\mathrm{Y}=\mathrm{a}+\mathrm{bX}$ where $\mathrm{a}$ and $\mathrm{b}$ are coefficients for each tooth type (Table 1) and $\mathrm{X}$ is the attachment level $(\mathrm{mm})$ defined as the distance from the cementoenamel junction to the tip of the inserted probe [1] (Figure 1). This formula was applied to derive a new index, the rPLI, as follows:

$$
\mathrm{rPLI}=\mathrm{nPLI} \times(\mathrm{Y} / 100)=\mathrm{nPLI} \times[(\mathrm{a}+\mathrm{bX}) / 100]
$$

Table 1. Root length, normal periodontal ligament index (nPLI), and residual periodontal ligament index (rPLI) values for each tooth type [5].

\begin{tabular}{|c|c|c|c|c|}
\hline & Code ${ }^{\mathrm{I}}$ & $\begin{array}{l}\text { Root length } \\
\text { (mm) }\end{array}$ & nPLI & rPLI \\
\hline \multicolumn{5}{|l|}{ Maxilla } \\
\hline Central incisor & U-CI & 12.2 & 2.6 & $2.6 \times(97.4-8.52 X) / 100$ \\
\hline Lateral incisor & U-LI & 13.4 & 2.6 & $2.6 \times(97.7-8.73 \mathrm{X}) / 100$ \\
\hline Canine & U-C & 16.6 & 3.8 & $3.8 \times(99.4-7.09 X) / 100$ \\
\hline First premolar & U-PM1 & 12.9 & 3.2 & $3.2 \times(98.2-8.53 \mathrm{X}) / 100$ \\
\hline Second premolar & U-PM2 & 13.9 & 3.0 & $3.0 \times(96.6-8.67 \mathrm{X}) / 100$ \\
\hline First molar & U-M1 & 13.5 & 6.0 & $6.0 \times(102.4-8.28 \mathrm{X}) / 100$ \\
\hline $\begin{array}{c}\text { Second molar } \\
\text { Mandible }\end{array}$ & U-M2 & 12.7 & 4.8 & $4.8 \times(99.8-8.49 \mathrm{X}) / 100$ \\
\hline Central incisor & L-CI & 12.0 & 2.1 & $2.1 \times(98.2-8.00 \mathrm{X}) / 100$ \\
\hline Lateral incisor & L-LI & 12.6 & 2.3 & $2.3 \times(98.9-8.90 \mathrm{X}) / 100$ \\
\hline Canine & $\mathrm{L}-\mathrm{C}$ & 14.9 & 3.4 & $3.4 \times(98.7-7.67 \mathrm{X}) / 100$ \\
\hline First premolar & L-PM1 & 14.7 & 3.1 & $3.1 \times(97.2-8.16 \mathrm{X}) / 100$ \\
\hline Second premolar & L-PM2 & 14.0 & 2.7 & $2.7 \times(96.5-8.56 \mathrm{X}) / 100$ \\
\hline First molar & L-M1 & 12.6 & 5.6 & $5.6 \times(100.7-7.99 X) / 100$ \\
\hline Second molar & L-M2 & 12.6 & 4.8 & $4.8 \times(98.9-8.42 \mathrm{X}) / 100$ \\
\hline
\end{tabular}

$\mathrm{rPLI}=(\mathrm{nPLI}) \times(\mathrm{a}+\mathrm{bX}) / 100 ; \mathrm{X}$ is the attachment level $(\mathrm{mm}) .(1 \leq \mathrm{X}<$ root length) The formula $(\mathrm{a}+\mathrm{bX})$ for calculating the residual periodontal ligament area percentage was described by Yamamoto et al. [4].

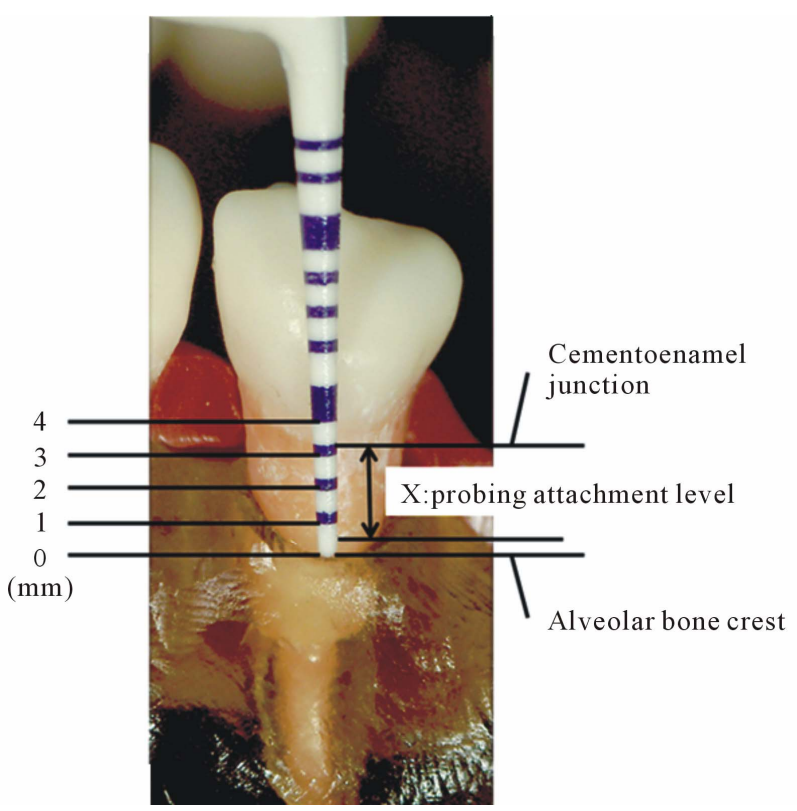

Figure 1. The attachment level $(\mathrm{X} ; \mathrm{mm})$ was determined by probing, with the value defined as the distance from the cementoenamel junction to the location of the inserted probe tip.

In this equation, nPLI is considered the initial index of the rPLI.

The length of the periodontal ligament attachment of the abutment tooth should be at least one-half to twothirds that of its normal attachment [3], and possible rPLI values were calculated.

\subsection{Occlusal Pressure for Each Tooth Type during Maximal Voluntary Clenching}

In a previous study [6], occlusal force, area and pressure for individual maxillary and mandibular teeth were analyzed by assessing their OSAs. The Ethics Committee for Epidemiologic Study of Hiroshima University approved the study protocol (No. 331), which followed the tenets of the Declaration of Helsinki. Ninety-nine subjects (49 men and 50 women; average age, $24.7 \pm 3.4$ years; range, 20 - 37 years) performed maximal voluntary clenching twice for $3 \mathrm{~s}$ in the intercuspal position with a pressure-sensitive sheet (thickness, $97 \mu \mathrm{m}$; dental prescale $50 \mathrm{H} / \mathrm{R}$ type, GC, Tokyo, Japan) placed between the maxillary and mandibular dental arch. Occlusal force, area and pressure of individual teeth were calculated by color development in the pressure-sensitive sheet with special analytical equipment (Occluzer 707, GC, Tokyo, Japan) and software (DePROS-PC709, GC, Tokyo, Japan). Occusal contact condition of individual teeth was confirmed using the intraocclusal record (EXABITE II, GC, Tokyo, Japan). All data were analyzed using unpaired Student's t-test, Kruskal-Wallis test and Scheffe's test for multiple comparisons with a significance level of $\mathrm{P}<0.05$. The occlusal pressure was adopted as the 
representative OSA for each individual tooth, although there were, in part, statistically significant differences in the effects of laterality and gender. Thus, we conclude that the occlusal pressure of individual teeth can be used as an indicator of OSA. In this study, the mean and $95 \%$ confidence interval $(\mathrm{CI})$ of occlusal pressure $(\mathrm{MPa})$ for individual teeth during maximal voluntary clenching for each nPLI value were used as a reference range of OSA for each tooth type.

\subsection{OSA Score}

The OSA score was calculated by the whole sum of nPLI or rPLI values of the remaining teeth. The OSA score obtained by the total nPLI values of the 28 teeth was 100 . The maximum and minimum OSA scores obtained using the total nPLI values of the remaining teeth corresponding to Eichner's classification were calculated. Moreover, the OSA scores obtained using the total nPLI values of the remaining teeth corresponding to Eichner's subclasses were calculated.

\section{RESULTS AND DISCUSSION}

\section{1. nPLI and rPLI of Each Tooth Type}

The nPLI and rPLI of each tooth type are shown in Table 1 and the rPLI values corresponding to one-half to two-thirds of its normal attachment length are shown in Table 2. For example, the nPLI value for $\mathrm{U}-\mathrm{C}$ is 3.8, and the rPLI value corresponding to one-half to two-thirds of its attachment length ranges from 1.6 to 2.4 . The clinical

Table 2. nPLI and rPLI values one-half to two-thirds of the length of the normal periodontal ligament attachment $[5,7]$.

\begin{tabular}{cccccc}
\hline \multirow{2}{*}{ Code } & \multirow{2}{*}{ nPLI } & \multicolumn{2}{c}{ One-half } & \multicolumn{2}{c}{ Two-thirds } \\
\cline { 3 - 6 } & & $\mathrm{X}(\mathrm{mm})$ & rPLI & $\mathrm{X}(\mathrm{mm})$ & rPLI \\
\hline Maxilla & & & & & \\
U-CI & 2.6 & 6 & 1.2 & 4 & 1.6 \\
U-LI & 2.6 & 6 & 1.2 & 4 & 1.6 \\
U-C & 3.8 & 8 & 1.6 & 5 & 2.4 \\
U-PM1 & 3.2 & 6 & 1.5 & 4 & 2.1 \\
U-PM2 & 3.0 & 6 & 1.3 & 4 & 1.9 \\
U-M1 & 6.0 & 6 & 3.2 & 4 & 4.2 \\
U-M2 & 4.8 & 6 & 2.3 & 4 & 3.1 \\
Mandible & & & & & \\
L-CI & 2.1 & 6 & 1.0 & 4 & 1.4 \\
L-LI & 2.3 & 6 & 1.1 & 4 & 1.5 \\
L-C & 3.4 & 7 & 1.5 & 4 & 2.3 \\
L-PM1 & 3.1 & 7 & 1.2 & 4 & 2.0 \\
L-PM2 & 2.7 & 7 & 1.0 & 4 & 1.7 \\
L-M1 & 5.6 & 6 & 3.0 & 4 & 3.8 \\
L-M2 & 4.8 & 6 & 2.3 & 4 & 3.1 \\
\hline X
\end{tabular}

$\mathrm{X}$ is the attachment level $(\mathrm{mm}) .(1 \leq \mathrm{X}<$ root length) implication of the difference between nPLI and rPLI has to be verified using in vitro simulation and epidemiologic data along with the OSA of each tooth type. Here, the suitability of the rPLI for prosthodontic treatment planning, such as selecting abutment teeth, establishing criteria for tooth extraction, choosing implants, and determining the optimal extent of denture base areas, was investigated.

\section{2. nPLI Values and Occlusal Pressure for Each Tooth Type during Maximal Voluntary Clenching}

The mean and 95\% confidence interval (CI) of occlusal pressure $(\mathrm{MPa})$ for each tooth type during maximal voluntary clenching [6] for each nPLI value is shown in Table 3 and Figure 2. The occlusal pressure increased gradually from CI (upper, 62.5; lower, 60.9 MPa), reached its peak at $\mathrm{C}$ (upper, 68.3; lower, 69.2 MPa) or PMI (upper, 68.3; lower, 67.8 MPa), and decreased sharply towards M2 (upper, 50.0; lower, 49.3 MPa). Moreover, the occlusal pressure of each individual tooth at M1 and M2 showed the opposite tendency to the nPLI. As the index corresponds to the normal periodontal ligament support and the occlusal pressure at M1 and M2 was smaller than the corresponding values at the other ipsilateral teeth, these findings may explain why molars show larger occlusal force than the other teeth. The greater periodontal ligament support of molars has an essential function because these teeth must crush and mash tough food effectively. Other mastication mecha-

Table 3. nPLI values and mean and $95 \%$ confidence interval (CI) occlusal pressure $(\mathrm{MPa})$ for each tooth type during maximal voluntary clenching [6].

\begin{tabular}{|c|c|c|c|}
\hline \multirow{2}{*}{ Code } & \multirow{2}{*}{ nPLI } & \multicolumn{2}{|c|}{ Occlusal pressure $(\mathrm{MPa})$} \\
\hline & & Mean & $95 \% \mathrm{CI}$ \\
\hline \multicolumn{4}{|l|}{ Maxilla } \\
\hline U-CI & 2.6 & 62.5 & $59.0-66.0$ \\
\hline U-LI & 2.6 & 65.6 & $60.3-70.9$ \\
\hline $\mathrm{U}-\mathrm{C}$ & 3.8 & 68.3 & $64.1-72.5$ \\
\hline U-PM1 & 3.2 & 68.3 & $64.2-72.4$ \\
\hline U-PM2 & 3.0 & 63.3 & $60.1-66.5$ \\
\hline U-M1 & 6.0 & 52.7 & $51.2-54.2$ \\
\hline U-M2 & 4.8 & 50.0 & $48.6-51.4$ \\
\hline \multicolumn{4}{|c|}{ Mandible } \\
\hline L-CI & 2.1 & 60.9 & $57.1-64.7$ \\
\hline L-LI & 2.3 & 67.5 & $62.3-72.7$ \\
\hline $\mathrm{L}-\mathrm{C}$ & 3.4 & 69.2 & $63.8-74.6$ \\
\hline L-PM1 & 3.1 & 67.8 & $63.6-72.0$ \\
\hline L-PM2 & 2.7 & 65.6 & $62.1-69.1$ \\
\hline L-M1 & 5.6 & 54.3 & $52.5-56.1$ \\
\hline L-M2 & 4.8 & 49.3 & $48.1-50.5$ \\
\hline
\end{tabular}




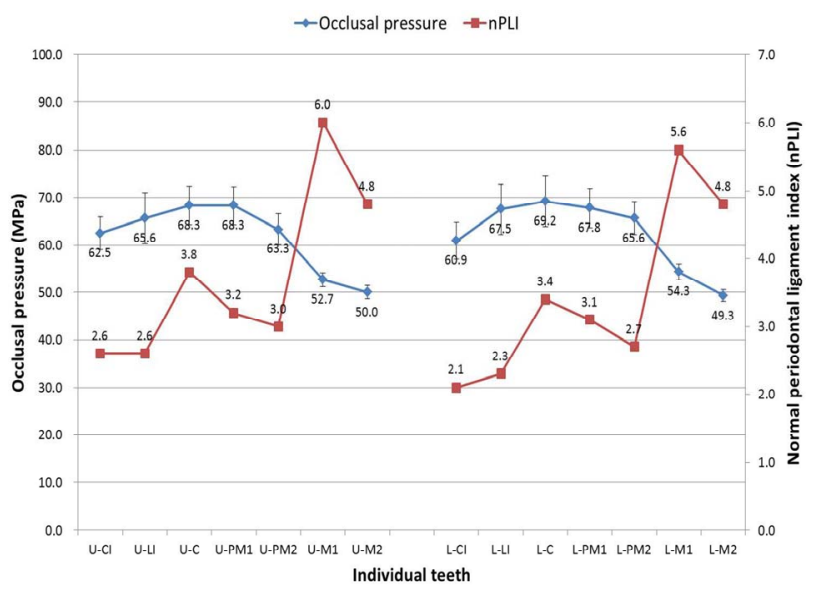

Figure 2. nPLI values and mean and 95\% confidence interval of occlusal pressure (MPa) for each tooth type from Table 3 [6]. The occlusal pressure increased gradually from CI, reached its peak at C or PMI, and decreased sharply towards M2. Abbreviations are as defined in Table 1.

nics further illuminate our study's findings. The anterior teeth or premolars guide jaw movement, and the premolars participate in the early stage of mastication, including moving the bolus of food towards the occlusal surface of the molars. The occlusal pressure from CI to PM2 was larger than that at M1 and M2, and so prostheses at the anterior or premolar teeth, in particular the maxilla, would bear a larger lateral force and run a greater risk of damage. Therefore, extremely close attention should be paid to the design of prostheses taking jaw position, movement, and parafunctional habits into consideration.

The OSA of each tooth type is not directly proportional to the status of residual periodontal ligament support. It is very important to evaluate the critical status of residual periodontal ligament support to maintain the OSA and clarify the relationship between nPLI (or rPLI) and the occlusal pressure of each tooth type.

\subsection{OSA Score}

The maximum and minimum OSA scores obtained using the total nPLI values of the remaining teeth corresponding to Eichner's classification are shown in Figure 3 [5]. The scores for classes A, B, and C were 30.6 - 100, 4.7 - 94.2, and 0.0 - 52.0, respectively. The OSA scores calculated using the total nPLI values of the remaining teeth corresponding to Eichner's subclasses are shown in Table 4 and were plotted in Figure 3 [7]. When the remaining teeth lost periodontal support, the OSA score calculated using the total rPLI value was lower than that calculated using the total nPLI. Moreover, the score calculated using the total nPLI value of a shortened dental arch comprising anterior and premolar teeth (i.e., subclass B2) required to fulfill the requirements of
Table 4. Occlusal-supporting ability (OSA) scores using the total nPLI of the remaining teeth corresponding to Eichner's subclasses [7].

\begin{tabular}{|c|c|c|c|c|c|}
\hline Subclasses & \multicolumn{4}{|c|}{ Remaining teeth } & $\begin{array}{l}\text { OSA } \\
\text { score }\end{array}$ \\
\hline \multirow{2}{*}{$\mathrm{A} 2$} & Maxilla & \multicolumn{2}{|c|}{$\begin{array}{lllllll}7 & 6 & 5 & 4 & 3 & 2 & 1\end{array}$} & 1234567 & \multirow{2}{*}{88.8} \\
\hline & Mandible & 7 & $\begin{array}{llll}5432 & 1\end{array}$ & $\begin{array}{llllll}1 & 2 & 3 & 4 & 5 & 7\end{array}$ & \\
\hline \multirow{2}{*}{$\mathrm{A} 3$} & Maxilla & 7 & 54321 & $\begin{array}{llllll}1 & 2 & 3 & 4 & 5 & 7\end{array}$ & \multirow{2}{*}{76.8} \\
\hline & Mandible & 7 & 543321 & $\begin{array}{llllll}1 & 2 & 3 & 4 & 5 & 7\end{array}$ & \\
\hline \multirow{2}{*}{ B1 } & Maxilla & 7 & 54321 & $\begin{array}{llllll}1 & 2 & 3 & 4 & 5 & 7\end{array}$ & \multirow{2}{*}{72.0} \\
\hline & Mandible & & $\begin{array}{llll}54321\end{array}$ & $\begin{array}{llllll}1 & 2 & 3 & 4 & 5 & 7\end{array}$ & \\
\hline \multirow{2}{*}{ B2 } & Maxilla & 7 & 54321 & $\begin{array}{llllll}1 & 2 & 3 & 4 & 5 & 7\end{array}$ & \multirow{2}{*}{67.2} \\
\hline & Mandible & & $\begin{array}{lllll}54321 & \end{array}$ & 12345 & \\
\hline \multirow{2}{*}{ B3 } & Maxilla & & 321 & 1234 & \multirow{2}{*}{43.0} \\
\hline & Mandible & & 4321 & 1234 & \\
\hline \multirow{2}{*}{ B4 } & Maxilla & & 321 & 123 & \multirow{2}{*}{33.6} \\
\hline & Mandible & & 321 & 123 & \\
\hline \multirow{2}{*}{$\mathrm{C} 1$} & Maxilla & & 3 & 3 & \multirow{2}{*}{16.4} \\
\hline & Mandible & & 21 & 12 & \\
\hline \multirow{2}{*}{$\mathrm{C} 2$} & Maxilla & & & & \multirow{2}{*}{15.6} \\
\hline & Mandible & & $\begin{array}{lll}321\end{array}$ & 123 & \\
\hline
\end{tabular}

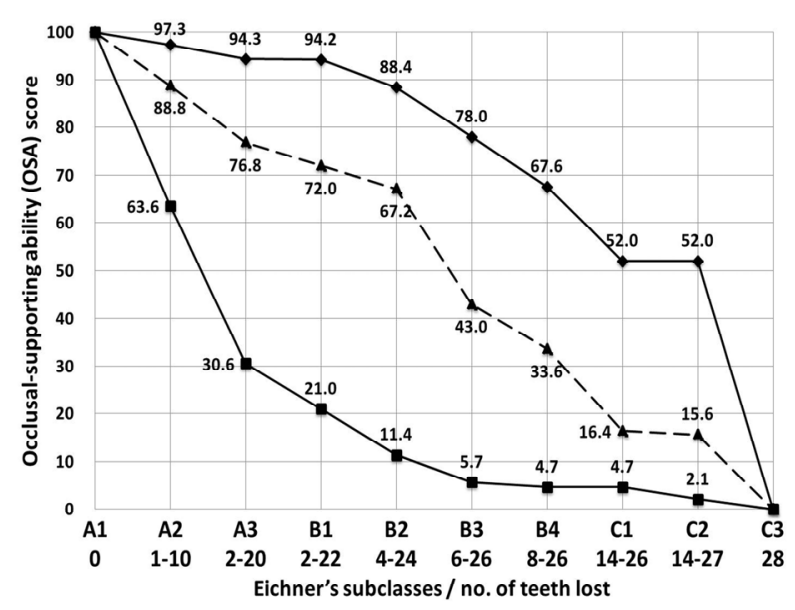

Figure 3. Maximum ( $\bullet$ ), minimum ( $\boldsymbol{\square})$, and example values (A) from Table 4 of occlusal-supporting ability scores for the remaining teeth corresponding to Eichner's classification $[5,7]$.

functional dentition is 57.7. Since first molar contacts are presumed to play an important role in oral health-related quality of life [9], the occlusal-supporting ability score of a dental arch comprising anterior, premolar, and first molar teeth (i.e. subclass A3) would be 81.0, which is approximately $40 \%$ higher than that for subclass B2. Thus, a score based on nPLI and rPLI is proposed as a suitable tool for epidemiologic research on the progression of tooth loss and the survival of prostheses. 


\section{ACKNOWLEDGEMENTS}

This study was supported in part by a Grant-in-Aid for Scientific Research (no. 23592847) from the Japan Society for the Promotion of Science (JSPS) and the Ministry of Education, Culture, Sports, Science and Technology (MEXT), Japan (2011-2013). We would like to thank Editage for providing editorial assistance.

\section{REFERENCES}

[1] Papapanou, P.N. and Lindhe, Y. (1997) Chapter 2 Epidemiology of periodontal disease. In: Lindhe, Y., Karring, T. and Lang, N.P., Eds., Clinical Periodontology and Implant Dentistry, 3rd Edition, Munksgaard, Copenhagen, 69-101.

[2] Johnston, J.F., Phillips, R.W. and Dykema, R.W. (1965) 1 Preoperative study. In: Johnston, J.F., Phillips, R.W. and Dykema, R.W., Eds., Modern Practice in Crown and Bridge Prosthodontics, 2nd Edition, W. B. Saunders Company, London, 3-18.

[3] Lulic, M., Brägger, U., Lang, N.P., Zwahlen, M. and Salvi, G.E. (2007) Ante's (1926) law revisited: A systematic review on survival rates and complications of fixed dental prostheses (FDPs) on severely reduced periodontal tissue support. Clinical Oral Implants Research, 18, 63-72. doi:10.1111/j.1600-0501.2007.01438.x
[4] Yamamoto, T., Kinoshita, Y., Tsuneishi, M., et al. (2006) Estimation of the remaining periodontal ligament from attachment-level measurements. Journal of Clinical Periodontology, 33, 221-225. doi:10.1111/j.1600-051X.2006.00888.x

[5] Abe, Y., Taji, T., Hiasa, K., Tsuga, K. and Akagawa, Y. (2010) A proposed index for residual periodontal ligament support. The International Journal of Prosthodontics, 23, 472-474.

[6] Abe, Y., Nogami, K., Mizumachi, W., Tsuka, H. and Hiasa, K. (2012) Occlusal-supporting ability of individual maxillary and mandibular teeth. Journal of Oral Rehabilitation, 39, 923-930. doi:10.1111/joor.12008

[7] Abe, Y., Taji, T., Hiasa, K., et al. (2010) Numerical occlusal support of remaining teeth using a residual periodontal ligament index (rPLI). The Journal of Hiroshima University Dental Society, 42, 1-8.

[8] Mizumachi, W., Abe, Y., Nogami, K., et al. (2010) Application of new occlusal support index to selection of abutment teeth of fixed partial dentures. The Journal of Hiroshima University Dental Society, 42, 151-154.

[9] Baba, K., Igarashi, Y., Nishiyama, A., et al. (2008) Patterns of missing occlusal units and oral health-related quality of life in SDA patients. Journal of Oral Rehabilitation, 35, 621-628. doi:10.1111/j.1365-2842.2007.01803.x 